\title{
Recent results from systematic parameterizations of Ginsparg-Wilson fermions
}

\author{
Christof Gattringer ${ }^{\mathrm{a} *}$ \\ a Institut für Theoretische Physik, Universität Regensburg, D-93040 Regensburg, Germany
}

The "Fixed Point Dirac Operator" and "Chirally Improved Fermions" both use large numbers of gauge paths and the full Dirac structure to approximate a solution of the Ginsparg-Wilson equation. After a brief review of the two approaches we present recent results for quenched QCD with pion masses down to $210 \mathrm{MeV}$. We discuss the limits and advantages of approximate parameterizations and outline future perspectives.

\section{Introduction}

The fundamental difficulties with the implementation of chiral symmetry on the lattice were finally overcome with the rediscovery of the Ginsparg-Wilson equation [1,2] for the lattice Dirac operator $D$,

$D \gamma_{5}+\gamma_{5} D=2 a D R \gamma_{5} D$.

On the right-hand side $R$ is a local operator commuting with $\gamma_{5}$ and $a$ denotes the lattice spacing. This equation implies exact chiral symmetry on the lattice [3]. Results obtained with Dirac operators obeying (11) exactly or approximately now allow to test QCD also in the chiral regime (see [ for a recent review). The most widely used chiral Dirac operator is the so-called overlap operator [5. The overlap formula gives a simple explicit prescription how to construct a Ginsparg-Wilson fermion (i.e. a solution of Eq. (11)) from any decent lattice Dirac operator. An approach related to the overlap formalism are domain wall fermions [6] where an auxiliary fifth dimension is used to implement the chiral symmetry.

Besides the overlap and domain wall fermions two more approaches to chiral symmetry are known, fixed point fermions [7, 8, [9, 10] and the chirally improved operator 11.12. The fixed point (FP) Dirac operator is constructed from the saddle point evaluation of the RG equations.

\footnotetext{
*The work reported here was done in the BGR (BernGraz-Regensburg) collaboration. The author is supported by the Austrian Academy of Sciences, APART 654.
}

The chirally improved (CI) operator is obtained by a systematic expansion of a solution of the Ginsparg-Wilson equation. In a practical application both the FP and the CI operator will use only finitely many terms (essentially restricted to the hypercube) and one can expect 13 only an approximation of a Ginsparg-Wilson fermion. Exact chiral symmetry can, however, be obtained by using the FP or CI operators as a starting point in the overlap projection (see also [14]). Using an improved operator for the overlap was found to improve the localization and one can hope to also obtain better dispersion and scaling properties. However, additional overlap steps also drive up the cost of the Dirac operator in numerical implementations.

In this contribution we analyze how far into the chiral region one can proceed with FP or CI fermions without additional overlap steps. Following a short review of the construction of FP and CI fermions we will discuss several recently obtained results. We address the spectrum of the Dirac operator and the determination of the topological charge and susceptibility from the index theorem. This is followed by a discussion of the results from quenched spectroscopy with emphasis on the pion mass close to the chiral limit where we extract the quenched chiral log parameter $\delta$. We study the scaling behavior of rho and proton masses and of the pion decay constant. We conclude with a discussion of the range of pion masses where one can work competitively with FP and 


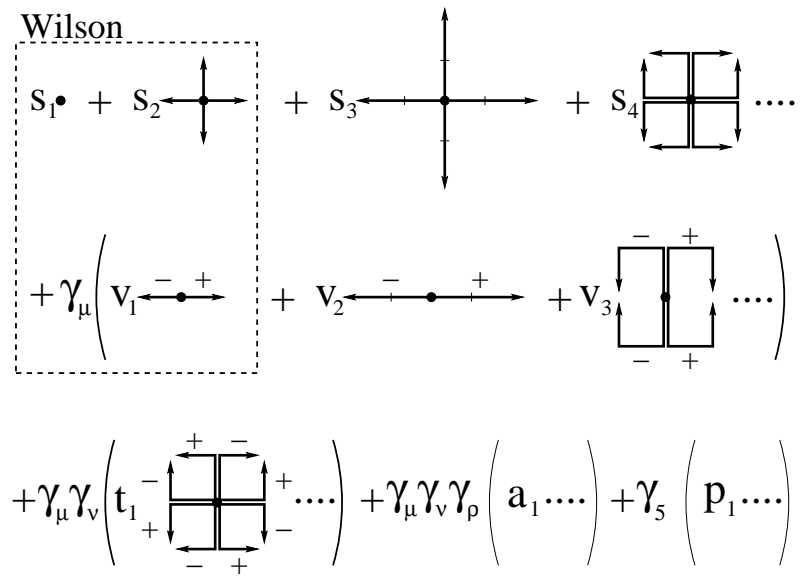

Figure 1. Schematic representation of a general lattice Dirac operator.

CI fermions without overlap projection.

\section{Construction of FP and CI fermions}

The construction for both the FP and CI operators starts from the expression for a general Dirac operator on the lattice 8, 11]. In Fig. 11 we give a schematic representation of such a general lattice Dirac operator.

Let us start the discussion of the structure with already familiar terms. In the dashed square in the upper left corner of Fig. 11 we show the terms that are used in the Wilson Dirac operator. There are two terms coming with the unit matrix in Dirac space: Firstly a constant term consisting of the mass parameter and the center term in the discretization of the Laplacian. They are represented by a dot. Secondly also the nearest neighbor terms from the discretization of the Laplacian come with a unit matrix in Dirac space. These hopping terms are represented by single hops, i.e. straight lines in all directions (for artistic reasons we show only two of the four possible directions). Besides the two terms trivial in Dirac space, the Wilson operator also contains the naively discretized massless Dirac operator where a sum over all $\gamma_{\mu}$ together with hops in positive and negative $\mu$-direction occurs (we show only one of the four directions). In order to de- scribe a derivative here the hops in positive and negative direction come with different signs. The parameters $s_{1}, s_{2}$ and $v_{1}$ are real numbers chosen such that a fermion free of doublers with a given mass is described.

A more general Dirac operator is obtained when more terms are used to discretize the derivative, such as next to nearest neighbor terms, staples etc. Similarly one can also extend the number of terms in the trivial Dirac sector. Finally from the Symanzik improvement program it is already known that also sectors of the Clifford algebra other than the trivial and vector sectors can contribute to the Dirac operator. So the structure of a general Dirac operator as depicted in Fig. 11 is a sum over all elements of the Clifford algebra. Each element is multiplied with paths of link variables and each path has some coefficient $s_{i}, v_{i}, t_{i}, a_{i}, p_{i}$. Some of the paths have the same coefficient but differ by relative sign factors. These sign factors are entirely determined by implementing the symmetries $\mathrm{C}, \mathrm{P}$ and $\gamma_{5}$-hermiticity (i.e. $\gamma_{5} D \gamma_{5}=D^{\dagger}$ ). Rotation invariance requires paths related by rotations on the lattice to come with the same coefficient and translation invariance makes the coefficients independent of the actual space time point. With our particular choice of the representation of the Clifford algebra the coefficients $s_{i}, v_{i}, t_{i}, a_{i}, p_{i}$ are real. A further generalization can be obtained by allowing these coefficients to be real functions of local loops of gauge links. This option is used for the construction of the FP Dirac operator.

The FP and the CI operator now differ only in the method for determining the coefficients $s_{i}, v_{i}, t_{i}, a_{i}, p_{i}$. For the FP operator the goal is to use the coefficients to approximate a solution of the fixed point equation for the Dirac operator. The basic idea is that near the critical surface the theory is invariant under real space renormalization group transformations that relate the quantum fields on a fine lattice to fields on a coarser lattice. In general the corresponding equations are quite involved but in the weak coupling limit they can be solved using the saddle point method. For the Dirac operator the FP equation reads

$D_{c}=\kappa-\kappa^{2} \Omega\left[D_{f}+\kappa \Omega^{\dagger} \Omega\right]^{-1} \Omega^{\dagger}$. 
Here $D_{c}\left(D_{f}\right)$ is the FP Dirac operator evaluated on the coarse (fine) gauge field configurations. $\Omega$ is the blocking kernel for the fermions and $\kappa$ a free parameter of the blocking procedure which can be used to maximize the slope of the exponential decay of the Dirac operator. The coefficients $s_{i}, v_{i}, t_{i}, a_{i}, p_{i}$ of the Dirac operator were now adjusted such that a $\chi^{2}$ functional measuring essentially the difference between the left-hand and right-hand sides of (2) on an ensemble of coarse and fine gauge configurations related by the renormalization group is minimized. In the actual construction this procedure was iterated starting from an ensemble at very weak coupling, followed by an intermediate step and finally a last minimization step at the target coupling. We remark that the action was optimized only for one such coupling corresponding to a lattice spacing of $0.16 \mathrm{fm}$ and then used also for the other two couplings. The parameterized FP operator is described by 82 couplings corresponding to 41 independent terms and each of the coefficients $s_{i}, v_{i}, t_{i}, a_{i}, p_{i}$ is a linear function of local closed gauge loops. All terms of the FP operator are restricted to the hypercube.

For the CI operator the strategy is to directly insert the expanded Dirac operator as depicted in Fig. 1 into the Ginsparg-Wilson equation (11) with a trivial kernel $R=1 / 2$. The evaluation of the two sides of Eq. (1) can be implemented in an algebraic computer program using directly the systematic representation of Fig. 11. The result is an expansion for both sides of the GinspargWilson equation similar to the expansion of the original Dirac operator. One can show that the individual terms (different element of the Clifford algebra, different paths) are linearly independent. The coefficients of equal terms on the two sides have to be equal and one can read off a system of coupled quadratic equations for the expansion coefficients

$$
\begin{aligned}
& 2 s_{1}=s_{1}^{2}+8 s_{2}^{2} \ldots+8 v_{1}^{2} \ldots \\
& 2 s_{2}=2 s_{1} s_{2}+12 s_{2} s_{3} \ldots+12 v_{1} v_{2} \ldots
\end{aligned}
$$$$
\text { ..... }
$$

This system is equivalent to the original Ginsparg-Wilson equation. After one truncates the expansion in Fig. 1 this system becomes finite and can be solved numerically. In addition to the equations representing the Ginsparg-Wilson equation one can impose boundary conditions, i.e. additional equations for the coefficients enforcing vanishing quark mass, the correct dispersion relation in the free case, $\mathcal{O}(a)$ improvement etc. The resulting CI operator is an approximation of a solution of the Ginsparg-Wilson equation. The CI operator has 19 coefficients and terms on the hypercube plus an extra L-shaped term of length $\sqrt{5}$.

\section{Spectrum of eigenvalues}

A first impression of the quality of the approximation of a Ginsparg-Wilson Dirac operator can be obtained by inspecting eigenvalues of the Dirac operator in typical gauge backgrounds. For an exact solution of the Ginsparg-Wilson equation the spectrum is restricted to a circle of radius 1 with center 1 in the complex plane. For the two approximations considered here the spectrum will not exactly lie on the Ginsparg-Wilson circle but show small fluctuations around it.

In Fig. 2 we show a superposition of 6 spectra of the CI operator in quenched background gauge configurations on $12^{3} \times 24$ lattices with lattice spacing $a=0.1 \mathrm{fm}$. Shown are the 50 smallest eigenvalues for each configuration. It is obvious that the eigenvalues are not located exactly on the Ginsparg-Wilson circle but the fluctuations are rather small. In particular we do not find many configurations with negative real eigenvalues. Such so-called exceptional configurations give rise to numerical problems in the computation of the propagator and limit the smallest quark masses one can work at. As will be discussed below the suppression of the eigenvalue fluctuations achieved by the FP and CI operators allows us to work at considerably smaller quark masses than with e.g. Wilson fermions.

An immediate consequence of the well ordered spectrum is the possibility to use the index theorem 15] for evaluating the topological charge with the FP and CI operators, while for Wilson fermions the large fluctuations of the eigenvalues lead to a mixture of physical and doubler modes 


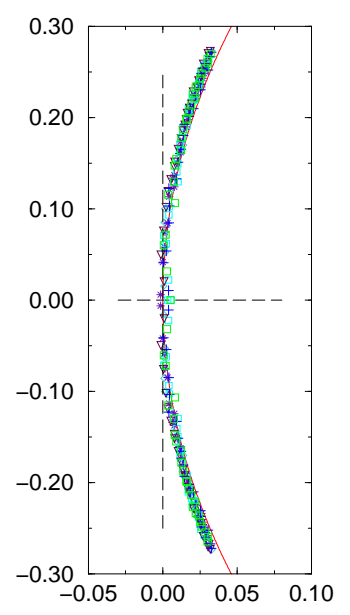

Figure 2. Superposition of 6 spectra of the CI operator. Shown are the 50 smallest eigenvalues in the complex plane for 6 configurations on $12^{3} \times$ 24 lattices with lattice spacing $a=0.1 \mathrm{fm}$.

[16]. We determined the topological charge as $\bar{Q}=n_{-}-n_{+}$where $n_{-}\left(n_{+}\right)$is the number of eigenvectors with negative (positive) matrix element with $\gamma_{5}$. Measurements [9, 17] on different volumes $V$ and lattice spacings give for the topological susceptibility $\left(\chi_{t o p}=\left\langle Q^{2}\right\rangle / V\right)$ values of $(196(4) \mathrm{MeV})^{4}$ for the FP operator and $(191(2) \mathrm{MeV})^{4}$ for the CI operator.

For the CI operator a detailed analysis of the behavior of $\chi_{t o p}$ across the finite temperature QCD phase transition was performed [17]. The quenched gauge configurations were generated with the Lüscher-Weisz gauge action [18]. Our results for the topological susceptibility as a function of the temperature are displayed in Fig. 3. One finds that the results obtained on $6 \times L^{3}$ lattices with spatial extent $L=12,16$ and 20 nicely agree with each other showing that finite size effects are under control. We also include results from $12^{4}$ and $16^{4}$ lattices to set the base line below $T_{c}$. The critical temperature $T_{c}$ as determined for the Lüscher-Weisz action in 19 is marked by the dashed vertical line. One finds that the topological susceptibility starts to decrease already below $T_{c}$ and quickly diminishes as the temperature increases further. The results obtained here using the index theorem agree well with calculations

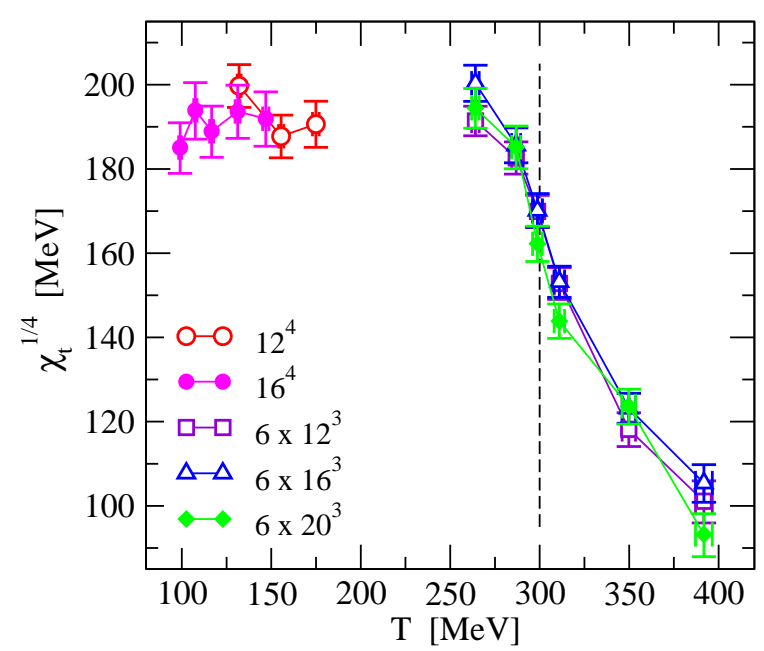

Figure 3. The behavior of the topological susceptibility across the QCD phase transition computed using the eigenvalues of the CI operator and the index theorem. The critical temperature is marked by a dashed vertical line.

based on bosonic methods for the determination of the topological charge [20].

\section{Quenched spectroscopy}

During the last year the major goal of the BGR collaboration was to use the FP and CI Dirac operators for quenched spectroscopy. Before we discuss the results of this study let us briefly outline the setting of these calculations.

For the FP operator the perfect gauge action 21] was used to generate the quenched ensembles. The gauge configurations were smoothened with perfect smearing [9] (which we consider as part of the parameterization of the Dirac operator) and subsequently fixed to Coulomb gauge. For the quark sources a Gaussian distribution was used.

The CI operator was used with gauge ensembles generated with the Lüscher-Weisz action [18]. Here the gauge configurations were treated with one step of hypercubic blocking [22]. For the CI operator Jacobi smeared sources [23] were used and no gauge fixing was necessary.

For both operators we worked on three lattice 


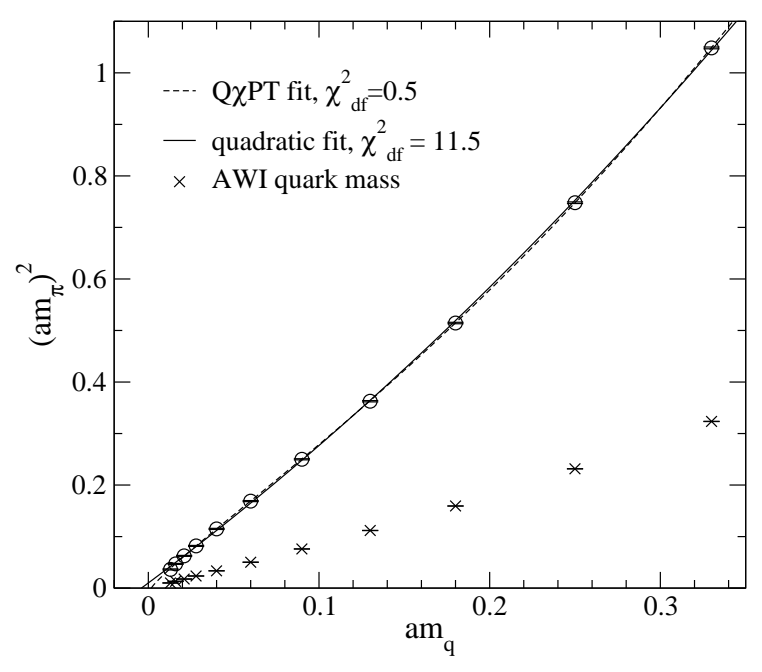

Figure 4. Pion mass (circles) and AWI mass (crosses) as a function of the bare quark mass. $16^{3} \times 32$ lattice, $a=0.16 \mathrm{fm}, \mathrm{FP}$ operator.

sizes, $8^{3} \times 24,12^{3} \times 24$ and $16^{3} \times 32$. For both gauge actions we used three different gauge couplings corresponding to lattice spacings of roughly $a=0.15$ ( 0.16 for FP), 0.10 and $0.08 \mathrm{fm}$ ( $a$ determined from the Sommer parameter). The statistics varied between 100 and 200 configurations for each ensemble. Our choice for the volumes and lattice spacings allows to study the scaling behavior with three different values of $a$ at a fixed spatial length of about $1.3 \mathrm{fm}$ and a finite size study at a fixed lattice spacing $a=0.16 \mathrm{fm}$. For a more detailed account of our setting for quenched spectroscopy see [24].

Let us begin the discussion of our quenched spectroscopy results with the pion mass and the axial Ward identity (AWI) mass. We computed the pion mass using different 2-point correlators (pseudoscalar, time component of the axial current, and the mixed correlator) and also compared correlators with smeared sink to point-like sink correlators. For all these measurements we find good agreement of the pion masses. The (unrenormalized) AWI mass $m_{A W I}$ was computed as

$m_{A W I}=\frac{\left\langle\partial_{0} A_{0} P\right\rangle}{2\langle P P\rangle}$,

where $P$ is the pseudoscalar density and $A_{0}$ the time component of the axial vector current. In Fig. 1 we show the mass of the pion as computed from the pseudoscalar 2-point function (circles) and the AWI mass (crosses) as a function of the bare quark mass. This plot was generated using the FP operator on a $16^{3} \times 32$ lattice at a lattice spacing $a=0.16 \mathrm{fm}$.

It is a convincing sign of good chiral properties that both the pion mass and the AWI mass extrapolate to zero with the bare quark mass. However, for smaller volumes ( $L=1.8$ or $1.3 \mathrm{fm}$ ) we found that the quenched topological finite size effects caused by the zero modes [25] become more important. They can be removed by subtracting the scalar propagator, which has the same topological finite size effect but larger mass, from the pseudoscalar 2-point function (see 24 for more details). In Fig. 1 we show also two fits to the pion data, a quadratic fit (full curve) and a fit including the quenched chiral log 26] (dashed curve). Our results for the quenched chiral log from a different method will be discussed below.

Another test of chirality is to determine the residual quark mass. This was done using a linear fit to the AWI mass and taking the intercept of the straight line with the horizontal axis as the residual quark mass $m_{\text {res }}$. For the FP operator we computed a residual mass of $a m_{r e s}=$ $-0.0006(4)$ at $a=0.16 \mathrm{fm}$ increasing in size to $a m_{\text {res }}=-0.0194(2)$ at $a=0.08$. This increase is due to the fact that we used the FP action which was optimized for the $a=0.16$ ensemble also at finer lattice spacing without redetermining the coefficients. For the CI operator the residual quark mass came out between $a m_{\text {res }}=0.002(1)$ at $a=0.15 \mathrm{fm}$ and $a m_{\text {res }}=0.000(1)$ at $a=0.08$ $\mathrm{fm}$. The smallest pion masses we have worked at are $m_{\pi}=210 \mathrm{MeV}$ for the FP operator and $m_{\pi}=240 \mathrm{MeV}$ for the CI operator. We expect that for both operators it is possible to go down to $m_{\pi} \sim 200 \mathrm{MeV}$ without having to use exceedingly fine lattices.

As another important benchmark measurement we looked at APE and Edinburgh plots. In Fig. 5 we show APE plots for the FP (top) and the CI (bottom) operator. For the FP operator we show three sets of data: The results for spatial extent $L=1.9 \mathrm{fm}$ at $a=0.16 \mathrm{fm}$ (circles), the same 

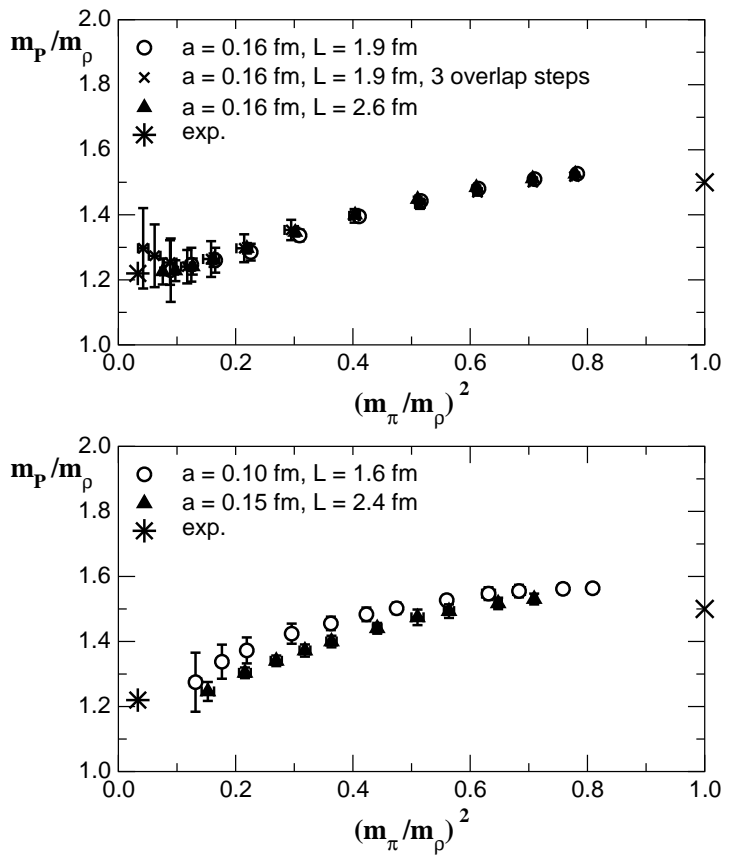

Figure 5. APE plots for the FP (top) and CI (bottom) operators.

ensemble but with the FP operator augmented with three steps of the overlap projection using Legendre polynomials (crosses) and finally results at $L=2.6 \mathrm{fm}, a=0.16 \mathrm{fm}$ (triangles). When comparing the FP operator on volumes with $L=$ $1.9 \mathrm{fm}$ and $L=2.6 \mathrm{fm}$ we find that the data agree well and we do not observe finite size effects for $L \geq 1.9 \mathrm{fm}$. Also the overlap augmented FP operator (crosses) gives rise to results which are in very good agreement with the unimproved FP data.

In the bottom figure for the CI operator we compare a data set from a finer but smaller lattice ( $a=0.1 \mathrm{fm}, L=1.6 \mathrm{fm}$, circles) to results from a lattice similar to the one used for the FP operator in the top figure $(a=0.15 \mathrm{fm}, L=2.4 \mathrm{fm}$, triangles). Here we do see a splitting between the two curves which we attribute to finite size effects and a small scaling violation. It is interesting to note that the data from the FP operator extrapolate very well to the physical value (marked by a star in the plot), while the result from the CI operator undershoots the physical value similar to

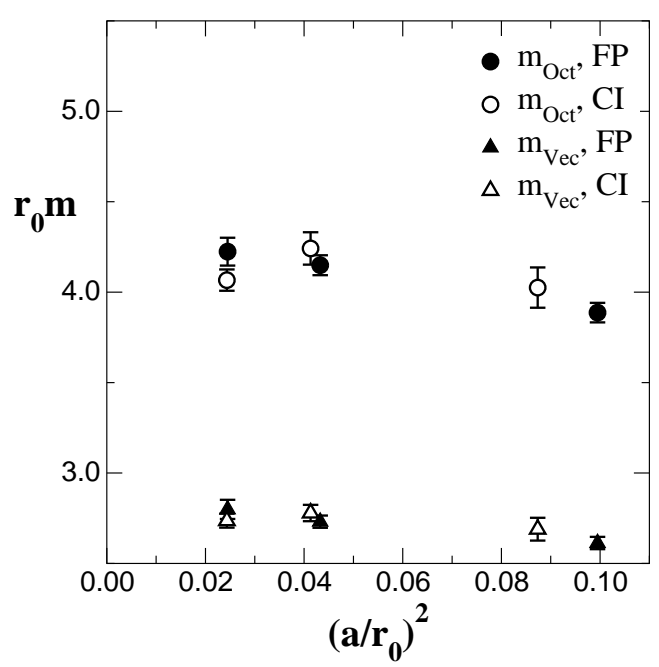

Figure 6. Scaling of the octet baryon mass (circles) and the vector mass (triangles). Filled symbols represent the FP results, while open symbols are used for the CI operator.

what is known from other quenched simulations (see e.g. 27]).

We conclude our discussion of the quenched spectroscopy with a brief discussion of the scaling properties of hadron masses. To do so, we work for both the FP and the CI operator at a bare quark mass which gives a ratio of $m_{\pi} / m_{\rho}=0.7$. At such a mass the statistical error is small and also a comparison with less chiral actions can be done (see e.g. [8]). In Fig. 6 we show the octet (circles) and vector (triangles) masses as a function of the square of the lattice spacing. For both the horizontal and vertical axes we use the Sommer parameter to set the scale. Filled symbols represent the FP results, while open symbols are used for the CI operator. The symbols are connected to guide the eye.

The results from the two operators agree within error bars. Both sets of data show only a small deviation from a horizontal line indicating that both $\mathcal{O}(a)$ and $\mathcal{O}\left(a^{2}\right)$ effects are small. Note that since both the parameterized FP operator as well as the CI operator are only approximate solutions of the Ginsparg-Wilson it can not a priori be expected that $\mathcal{O}(a)$ effects are absent. However, the 


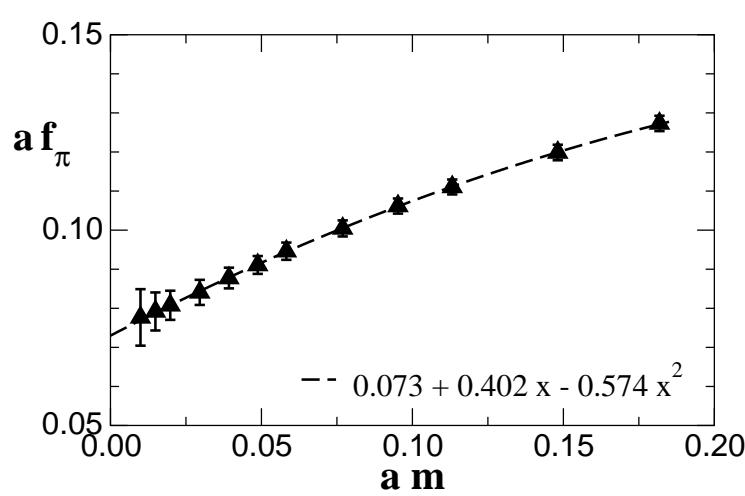

Figure 7. Pion decay constant as a function of the bare quark mass. $16^{3} \times 32$ lattice, $a=0.1 \mathrm{fm}$, CI operator.

plot shows that scaling violations are very small.

\section{Pion decay constant}

The definition of the pion decay constant can be combined with the axial Ward identity to yield

$f_{\pi}=\frac{2 m \sqrt{Z_{P P}}}{m_{\pi}^{2}}$.

Here $Z_{P P}$ is the prefactor of the pseudoscalar 2point function at zero-momentum,

$\sum_{x}\langle P(x, t) P(0,0)\rangle \sim \frac{Z_{P P}}{2 m_{\pi}} \exp \left(-m_{\pi} t\right)$.

Strictly speaking formula (5) holds only for Dirac operators with exact chiral symmetry where the product of renormalization constants $Z_{m} Z_{P}$ equals 1. For the approximate solutions of the Ginsparg-Wilson equation which we use here this is currently only an assumption which will eventually have to be tested.

In Fig. 7 we show $f_{\pi}$ according to Eq. (5) as a function of the bare quark mass (both in units of the lattice spacing as determined from the Sommer parameter) for the CI operator on the $16^{3} \times 32$ ensemble at $a=0.1 \mathrm{fm}$. We interpolate the data using a second order polynomial which we subsequently use to extrapolate the data to the chiral limit. We collected the results from the chirally extrapolated values of $f_{\pi}$ from the CI operator and analyzed the scaling of this observable. We find that all data agree well within error bars and the discretization errors are small. Our quenched results come out 10-15\% larger than the experimental value (see 24] for a more detailed discussion).

\section{Quenched chiral logs}

We have already briefly addressed the possibility of extracting the quenched chiral log parameter $\delta$ from the pion mass (compare Fig. 1 and the discussion below the figure). However, here we use a method 27 which allows to increase the statistics by using pions with non-degenerate quark masses and which also gets rid of the dependence on the unknown chiral perturbation scale $\Lambda_{\chi}$.

The idea is to form the combinations $x$ and $y$

$$
\begin{aligned}
& x=2+\frac{m_{1}+m_{2}}{m_{1}-m_{2}} \ln \left(\frac{m_{2}}{m_{1}}\right), \\
& y=\frac{4 m_{1} m_{2}}{\left(m_{1}+m_{2}\right)^{2}} \frac{M_{12}{ }^{4}}{M_{11}{ }^{2} M_{22}{ }^{2}} .
\end{aligned}
$$

Here $M_{12}$ denotes the mass of the pseudoscalar with quark masses $m_{1}$ and $m_{2}$. Using the results of [26] one finds

$y=1+\delta x+\mathcal{O}\left(m^{2}, \delta^{2}, a_{\phi}\right)$,

i.e. to leading order the quenched chiral log parameter can be read off from the slope in a plot of $y$ versus $x$.

In Fig. 8 we show such a $x-y$ plot for the FP operator on the $16^{3} \times 32$ lattice at $a=0.16 \mathrm{fm}$. The data lie inside a band which gives rise to $\delta=0.17(2)$ (compare 28] for previous determinations of $\delta$ with chiral fermions). For the CI operator we obtain a very similar result of $\delta=0.18(3)$. These results were obtained by using the unrenormalized AWI quark mass for $m_{1}, m_{2}$ in Eq. (7). For the CI operator we have also experimented with using the bare quark mass instead and we present a more detailed discussion in 24].

We remark that we also have obtained preliminary results for the pion scattering lengths and some details are also presented in [24]. 


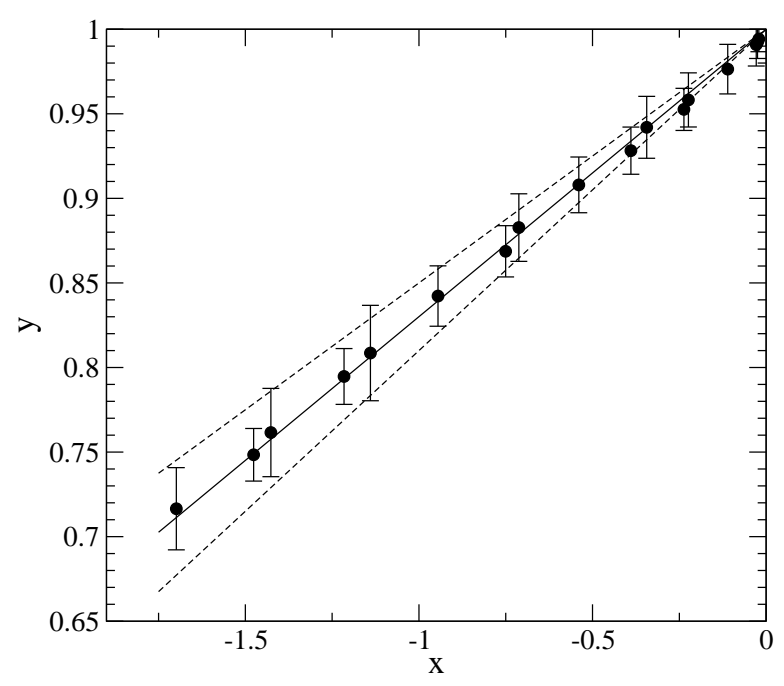

Figure 8. $x-y$ plot used to determine the quenched chiral $\log$ parameter $\delta$ (FP operator, $16^{3} \times 32, a=$ $0.16 \mathrm{fm})$.

\section{Conclusions}

In this contribution we have presented results from quenched QCD calculations using the parameterized FP and CI operators. Both these operators are approximate solutions of the Ginsparg-Wilson equation and are expected to have good chiral properties. Here these expectations are tested using various observables.

We find that both the pion and the AWI mass nicely extrapolate to 0 as a function of the bare quark mass. If one uses the offset in a linear extrapolation of the AWI mass as a criterion for the remaining chiral symmetry breaking we find residual quark masses between 1 and $4 \mathrm{MeV}$ on a quite coarse lattice with $a=0.16 \mathrm{fm}$. We have successfully extracted the quenched chiral $\log$ parameter $\delta$ using pions with non-degenerate quark masses. The octet and vector masses, as well as the pion decay constant show very good scaling behavior. We have demonstrated that we can reach pion masses of about $210 \mathrm{MeV}$ without having to go to very small lattice spacings. A more detailed account of our measurements can be found in [24].

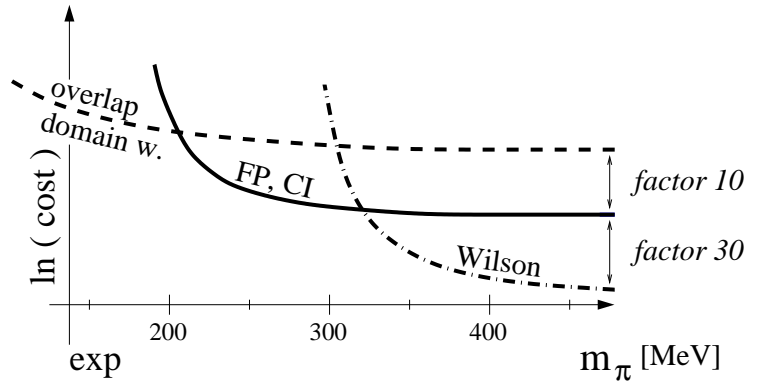

Figure 9. A sketch comparing the cost of different Dirac operators as a function of the pion mass.

We would like to wrap up our conclusions with a brief comment on where we think our approximate Ginsparg-Wilson fermions will be competitive. In Fig. 9 we show a sketch which compares the cost of different lattice Dirac operators as a function of the pion mass. For heavy quarks the operator of choice is certainly Wilson's Dirac operator, maybe combined with clover improvement. However, if one wants to go to smaller masses one has to increase the inverse gauge coupling to reduce eigenvalue fluctuations responsible for exceptional configurations. This leads to a finer lattice and the volume has to be increased which drives up the cost, making Wilson fermions very expensive below pion masses of $300 \mathrm{MeV}$. At such small masses it is more economical to use the more expensive FP or CI operators which cost about a factor of 30 more than Wilson's operator (see [24] for a more detailed discussion of the cost). As already remarked, with these operators we reached $210 \mathrm{MeV}$ without having to go to very fine lattices and we expect that we will remain competitive down to at least $200 \mathrm{MeV}$. If one needs to go further into the chiral region, e.g. when computing the chiral condensate, one eventually has to use overlap projection to further decrease the pion mass. We believe that in the window between pion masses of 200 and $300 \mathrm{MeV}$ the FP and CI operators are the best choice and we will explore whether this is sufficient to make reliable contact with chiral perturbation theory. 
Acknowledgements: I would like to thank the members of the BGR collaboration for sharing their experience and enthusiasm during this first year of work. The calculations were done on the Hitachi SR8000 at the Leibniz Rechenzentrum in Munich and we thank the LRZ staff for training and support. This work was supported in parts by DFG and BMBF.

\section{REFERENCES}

1. P. Ginsparg, K.G. Wilson, Phys. Rev. D 25 (1982) 2649.

2. P. Hasenfratz, Nucl. Phys. Proc. Suppl. 63 (1998) 53.

3. M. Lüscher, Phys. Lett. B 428 (1998) 342.

4. L. Giusti, Exact chiral symmetry on the lattice: $Q C D$ applications, plenary talk at Lattice' 02 , these proceedings.

5. R. Narayanan and H. Neuberger, Phys. Lett. B 302 (1993) 62, Nucl. Phys. B 443 (1995) 305.

6. D.B. Kaplan, Phys. Lett. B 288 (1992) 342; Y. Shamir, Nucl. Phys. B 406 (1993) 90; V. Furman and Y. Shamir, Nucl. Phys. B 439 (1995) 54.

7. P. Hasenfratz, F. Niedermayer, Nucl. Phys. B 414 (1994) 785.

8. P. Hasenfratz, S. Hauswirth, K. Holland, T. Jörg, F. Niedermayer and U. Wenger, Int. J. Mod. Phys. C 12 (2001) 691.

9. S. Hauswirth, Light hadron spectroscopy in quenched lattice $Q C D$ with chiral fixed-point fermions, Thesis, Bern University 2002, heplat/0204015; T. Jörg, Chiral measurements in quenched lattice $Q C D$ with fixed-point fermions, Thesis, Bern University 2002, heplat/0206025.

10. P. Hasenfratz, S. Hauswirth, T. Jörg, F. Niedermayer and K. Holland, hep-lat/0205010.

11. C. Gattringer, Phys. Rev. D 63 (2001) 114501.

12. C. Gattringer, I. Hip, C.B. Lang, Nucl. Phys. B 597 (2001) 451.

13. I. Horváth, Phys. Rev. Lett. 81 (1998) 4063, Phys. Rev. D 60 (1999) 034510; W. Bietenholz, hep-lat/9901005.

14. W. Bietenholz and I. Hip, Nucl. Phys. B
570 (2000) 423; T. DeGrand, Phys. Rev. D 63 (2001) 034503; W. Bietenholz, heplat/0204016.

15. P. Hasenfratz, V. Laliena and F. Niedermayer, Phys. Lett. B 427 (1998) 125.

16. C. Gattringer and I. Hip, Nucl. Phys. B 536 (1998) 363, Nucl. Phys. Proc. Suppl. 73 (1999) 871.

17. C. Gattringer, R. Hoffmann and S. Schaefer, Phys. Lett. B 535 (2002) 358.

18. M. Lüscher and P. Weisz, Commun. Math. Phys. 97 (1985) 59; Err.: 98 (1985) 433; G. Curci, P. Menotti and G. Paffuti, Phys. Lett. B 130 (1983) 205, Err.: B 135 (1984) 516.

19. C. Gattringer, P.E.L. Rakow, A. Schäfer and W. Söldner, Phys. Rev. D (in print), heplat/0202009.

20. B. Alles, M. D'Elia and A. Di Giacomo, Nucl. Phys. B 494 (1997) 281.

21. F. Niedermayer, P. Rüfenacht and U. Wenger, NPB, 2001. Nucl. Phys. B 597 (2001) 413.

22. A. Hasenfratz and F. Knechtli, Phys. Rev. D 64 (2001) 034504.

23. C. Best, M. Göckeler, R. Horsley, E.M. Ilgenfritz, H. Perlt, P.E.L. Rakow, A. Schäfer, G. Schierholz, A. Schiller and S. Schramm Phys. Rev. D 56 (1997) 2743; C. R. Allton et al. (UKQCD Collaboration), Phys. Rev. D 47 (1993) 5128.

24. C. Gattringer et al. (BGR collaboration), these proceedings, hep-lat/0209099.

25. T. Blum et al., hep-lat/0007038; S.J. Dong, T. Draper, I. Horváth, F.X. Lee, K.F. Liu and J.B. Zhang, Phys. Rev. D 65 (2002) 054507.

26. S.R. Sharpe, Phys. Rev. D 46 (1992) 3146; C.W. Bernard and M.F.L. Golterman, Phys. Rev. D 46 (1992) 853.

27. S. Aoki et al. (CP-PACS collaboration), heplat/0206009.

28. T. W. Chiu and T. H. Hsieh, Phys. Rev. D 66 (2002) 014506; T. Draper, S.J. Dong, I. Horváth, F.X. Lee, K.F. Liu, N. Mathur and J.B. Zhang, hep-lat/0208045. 